\title{
Neurobiologie
}

\section{Molekulare Kontrolle der Stabilität und Plastizität von Synapsen}

ZEESHAN MUSHTAQ, JAN PIELAGE

ABTEILUNG FÜR ZOOLOGIE UND NEUROBIOLOGIE, TU KAISERSLAUTERN

The precise regulation of synaptic connectivity is essential for the processing of information in the brain. Any aberrant loss of synaptic connectivity due to genetic mutations will disrupt information flow in the nervous system and may represent the underlying cause of psychiatric or neurodegenerative diseases. Therefore, identification of the molecular mechanisms controlling synaptic plasticity and maintenance is essential for our understanding of neuronal circuits in development and disease.

DOI: $10.1007 / \mathrm{s} 12268-021-1639-8$

(c) Die Autoren 2021

Das Erzeugen von Verhaltensmustern bei Tieren basiert auf neuronalen Schaltkreisen, die sensorische Informationen wahrnehmen, integrieren und in motorische Signale umsetzen. Zentrales Element dieser Schaltkreise sind synaptische Verbindungen zwischen Neuronen im zentralen Nervensystem bzw. zwischen Neuronen und Muskeln im peripheren Nervensystem. Für die Verarbeitung

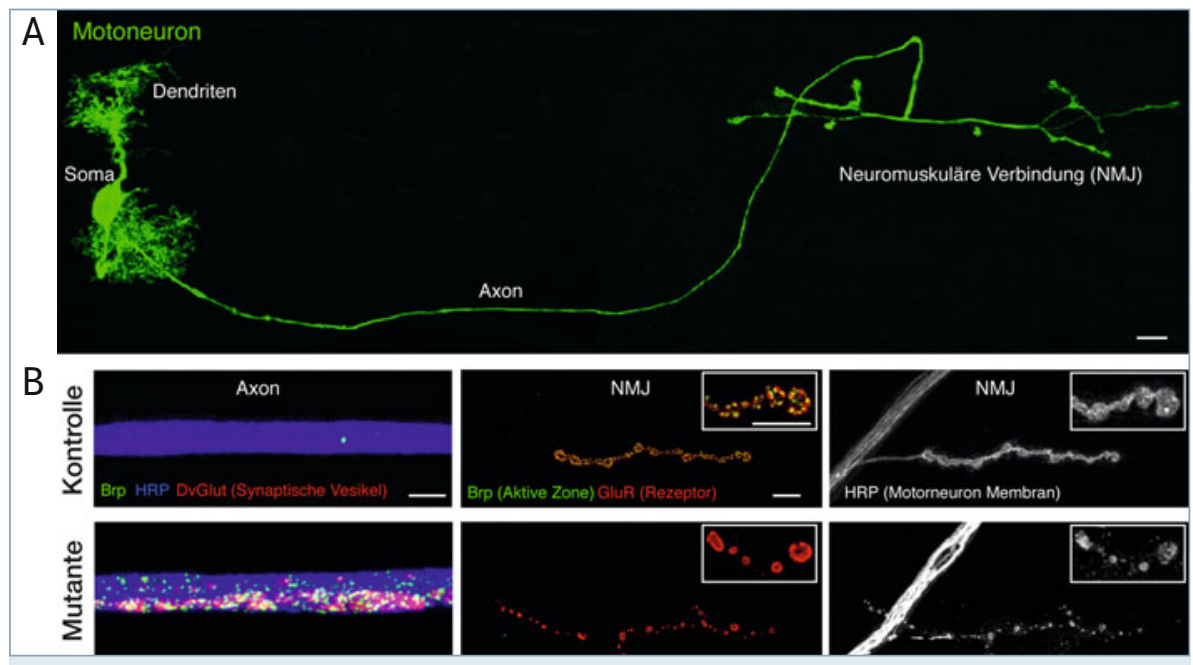

Abb.1: Drosophila-Motoneurone als Modellsystem für synaptische Stabilität und Plastizität. A, Die genetische Markierung eines einzelnen Motoneurons erlaubt die gleichzeitige Analyse aller neuronaler Kompartimente. B, Die vergleichende Analyse zeigt Defekte in axonalem Transport und synaptischer Stabilität. Es kommt zu einem Verlust von Proteinen der aktiven Zone (Bruchpilot, Brp) und einer Fragmentierung der synaptischen Membran. Maßstab: $10 \mu \mathrm{m}$. der axonalen Wegfindung [1] und der Synapsenbildung [2]. Für die Aufrechterhaltung der Funktion des Nervensystems ist es entscheidend, dass synaptische Verbindungen über lange Zeit stabil gehalten werden können. Diese Stabilität bezieht sich sowohl auf funktionelle als auch strukturelle Aspekte zentraler und peripherer Synapsen.

Genetische Mutationen, die zu einem Verlust synaptischer Stabilität führen, sind per se potenzielle Ursachen progressiver neurodegenerativer oder neuropsychiatrischer Erkrankungen, da der Informationsfluss innerhalb des Nervensystems nicht mehr aufrechterhalten werden kann. Der Verlust synaptischer Konnektivität ist eines der ersten Kennzeichen nahezu aller neurodegenerativer Erkrankungen des Menschen, wie z. B. bei der amyotrophen Lateralsklerose (ALS), der Alzheimer-Krankheit (AD) oder anderer progressiver Neuropathien [3]. Einhergehend mit dieser Symptomatik des Verlusts synaptischer Konnektivität werden häufig Störungen des axonalen Transports beobachtet. Hierbei ist zurzeit häufig noch unklar, ob Defekte des axonalen Transports ursächlich oder sekundäre Konsequenzen der Erkrankung sind [4].

\section{Drosophila als Modellsystem für} neurodegenerative Erkrankungen Für die Herstellung kausaler Zusammenhänge zwischen Beeinträchtigungen des axonalen Transports und synaptischen Defekten ist es essenziell, dass beide Phänotypen gleichzeitig analysiert werden können. Eine solche Analyse gestaltet sich in komplexen Nervensystemen der Vertebraten oft schwierig. Die Verwendung von Drosophila-Motoneuronen als Modellsystem hingegen erlaubt eine simultane Analyse axonaler und synaptischer Phänotypen und ermöglicht eine präzise Beschreibung und Quantifizierung struktureller und funktioneller synaptischer Defekte. Die larvalen Motoneurone haben komplexe Dendritenbäume innerhalb des zentralen Nervensystems und senden ein Axon zu den peripheren Muskeln, um dort 
elaborierte neuromuskuläre Verbindungen $\mathrm{zu}$ bilden (neuromuscular junction, NMJ; Abb. 1 ).

Innerhalb der NMJ ist die Visualisierung aller individueller Synapsen durch eine KoFärbung mit dem präsynaptischen aktiven Zonen-Marker Bruchpilot (Brp) und postsynaptischen Glutamatrezeptoren (GluR) möglich (Abb. 1B, Abb. 2D). Gleichzeitig kann die Morphologie der NMJ durch eine Färbung der Motoneuronmembran analysiert werden (Abb. 1B, Kontrolle). Defekte in der Stabilität der synaptischen Verbindungen zeigen sich als ein Verlust der Apposition der beiden synaptischen Marker, da der Abbau der postsynaptischen Glutamatrezeptoren erst mit deutlicher zeitlicher Verzögerung stattfindet [5]. Zugleich kommt es zu einer Fragmentierung der Zellmembran des Motoneurons (Abb. 1B, Mutante).

Die synaptische Degeneration beginnt zumeist am terminalen synaptischen Bouton und betrifft dann progressiv weitere Bereiche der neuromuskulären Verbindung, bis es zu einem kompletten Verlust der Muskelinnervation kommt. Diese Phänotypen konnten bisher u. a. bei Mutationen in Zelladhäsionsmolekülen, Scaffold-, Cytoskelettkomponenten und Regulatoren sowie in Signaltransduktionsmolekülen beobachtet werden, die die Koordination zwischen Prä- und Postsynapse regulieren [5-7]. Interessanterweise kommt es auch in Drosophila-Motoneuronen zu einem gleichzeitigen Auftreten von axonalen Transportdefekten (Abb. 1B, unteres Panel, [5]). Hervorzuheben ist, dass die grundlegenden zellbiologischen Mechanismen zwischen Fliege und Menschen konserviert sind, da Mutationen in korrespondierenden Genen bei Mausmodellen und beim Menschen zu Neurodegeneration und psychiatrischen Defekten führen [8]. Die Kombination von gezielten genetischen Manipulationen mit in vivo-Mikroskopie dieses einfach zugänglichen Systems bietet nun die Möglichkeit, die molekularen und zellulären Mechanismen der zugrunde liegenden Prozesse aufzuklären. So können genetische
Eingriffsmöglichkeiten definiert werden, die anschließend im Mausmodell auf ihr therapeutisches Potenzial getestet werden können. Ein interessanter Ansatzpunkt ist z. B. die Verbesserung der funktionellen Plastizität, da kürzlich ein direkter Zusammenhang zwischen der Regulation der präsynaptischen Homöostase und synaptische Degeneration im Fliegen und Mausmodell nachgewiesen werden konnte [9].

\section{Analyse komplexer Schaltkreise für Geschmackserkennung, Lernen und Gedächtnis}

Zur Aufklärung der grundlegenden Mechanismen der synaptischen Plastizität ist das neuromuskuläre System von Drosophila prädestiniert, da hier die Struktur und Funktion einzelner synaptischer Verbindungen mithilfe von Super-Resolution-Mikroskopie (stimulated emission depletion, STED-Mikroskopie, Abb. 2D) möglich ist und zugleich mithilfe elektrophysiologischer Ableitungen die funktionellen Parameter bestimmt werden kön-

\title{
Hier steht eine Anzeige.
}

\author{
望 Springer
}



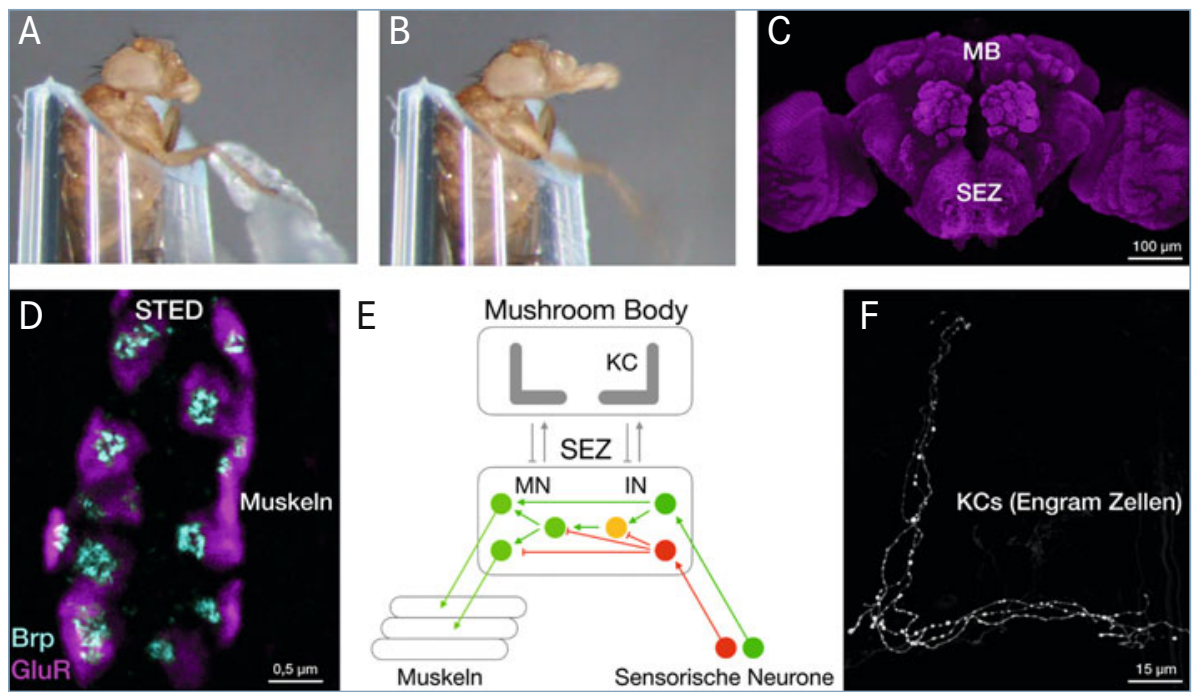

$\Delta$ Abb. 2: Zelluläre und molekulare Kontrolle komplexer Verhaltensmuster. A-B, Stimulation von Geschmacksrezeptoren an den Füßen der Fliege löst ein stereotypes Ausstrecken des Rüssels aus. C, das adulte Gehirn. Die Bereiche des Geschmacksschaltkreises (SEZ) und des Gedächtnisses (MB) sind markiert. D-F, neuronale Kontrolle der Geschmacksantwort und des Gedächtnisses. Sensorische Neurone leiten positive (grün) und negative (rot) Geschmackseindrücke an Interneurone (IN) in der subösophagealen Zone (SEZ). Dort wird das Signal prozessiert und an Motoneurone (MN) weitergeleitet (E). Ziel ist ein Verständnis der molekularen Mechanismen der Bewegungskontrolle mithilfe von Super-Resolution-Mikroskopie (STED, D) und anhand genetischer Markierung von Gedächtniszellen (F).

nen. Für unser Verständnis komplexer Informationsverarbeitung im Gehirn ist eine Übertragung der gewonnenen Erkenntnisse sowohl auf einfache als auch auf komplexe neuronale Schaltkreise notwendig. Die kürzliche Erstellung des kompletten synaptischen Konnektoms großer Teile des adulten Gehirns von Drosophila [10] ermöglicht nun eine systematische Analyse der Zusammenhänge zwischen synaptischer Funktion und Verhalten. Beispiel für einen sensorischmotorischen Schaltkreis ist die Antwort der Fliege auf einen positiven Geschmacksstimulus. Die Aktivierung gustatorischer sensorischer Neurone an den Beinen der Fliege durch Zucker löst in Tieren stereotypisches Verhalten aus; die Fliege streckt ihren Rüssel in Richtung der Futterquelle (Abb. 2A, B). Diese Antwort stellt keinen einfachen Reflex dar, sondern wird nur nach Abgleich mit dem internen Zustand der Fliege (z. B. Hunger) ausgelöst. Hierfür verantwortlich sind wenige Neurone der 100.000 Neurone des Fliegengehirns innerhalb des subösophagealen Zentrums (SEZ) (Abb. 2C, E). Diese Neurone können selektiv aktiviert oder inhibiert werden, um so ihre Funktion innerhalb des Schaltkreises aufzuklären [11].

Das synaptische Konnektom des Gedächtniszentrums der Fliege ermöglicht nun, zusammen mit der Möglichkeit Gedächtnisneurone genetisch zu markieren und mani- pulieren (Abb. 2F, [12]), auch die molekularen und zellulären Grundlagen der Gedächtnisbildung zu erforschen. Hier kann die Funktion von Kandidatengenen, die die strukturelle oder funktionelle Plastizität einzelner synaptischer Verbindungen an der NMJ beeinflussen, gezielt für Lernen und Gedächtnis getestet werden. Diese korrelative Analyse genetischer Funktionen an einzelnen Synapsen und innerhalb komplexer Schaltkreise erweitert so unsere Kenntnisse der normalen Entwicklung des Nervensystems und ermöglicht zugleich neue Einblicke in die Entstehung und Behandlung neurodegenerativer Erkrankungen.

\section{Literatur}

[1] Evans TA, Bashaw GJ (2010) Axon guidance at the midline: of mice and flies. Curr Opin Neurobiol 20: 79-85 [2] Van Vactor D, Sigrist SJ (2017) Presynaptic morphogenesis, active zone organization and structural plasticity in Drosophila. Curr Opin Neurobiol 43: 119-129

[3] Yaron A, Schuldiner O (2016) Common and divergent mechanisms in developmental neuronal remodeling and dying back neurodegeneration. Curr Biol 26: R628-R639 [4] Holzbaur ELF, Scherer SS (2011) Microtubules, axonal transport, and neuropathy. N Engl J Med 365: 2330-2332 [5] Stephan R, Goellner B, Moreno E et al. (2015) Hierarchical microtubule organization controls axon caliber and transport and determines synaptic structure and stability. Dev Cell 33: 5-21

[6] Enneking E-M, Kudumala SR, Moreno E et al. (2013) Transsynaptic coordination of synaptic growth, function, and stability by the L1-type CAM Neuroglian. PLoS Biol 11: e1001537

[7] Bulat V, Rast M, Pielage J (2014) Presynaptic CK2 promotes synapse organization and stability by targeting Ankyrin2. J Cell Biol 204: 77-94

[8] Smith KR, Kopeikina KJ, Fawcett-Patel JM et al. (2014) Psychiatric risk factor ANK3/ankyrin-G nanodomains regulate the structure and function of glutamatergic synapses. Neuron 84: 399-415

[9] Orr BO, Hauswirth AG, Celona B et al. (2020) Presynaptic homeostasis opposes disease progression in mouse models of ALS-like degeneration: evidence for homeostatic neuroprotection. Neuron 107: 95-111

[10] Takemura SY, Aso Y, Hige T et al. (2017) A connectome of a learning and memory center in the adult Drosophila brain. Elife 6: e26975

[11] Schwarz 0, Bohra AA, Liu X et al. (2017) Motor control of Drosophila feeding behavior. Elife 6: e19892

[12] Siegenthaler D, Escribano B, Bräuler V et al. (2019) Selective suppression and recall of long-term memories in Drosophila. PLoS Biol 17: e3000400

Funding note: Open Access funding enabled and organized by Projekt DEAL Open Access: Dieser Artikel wird unter der Creative Commons Namensnennung 4.0 International Lizenz veröffentlicht, welche die Nutzung, Vervielfälttigung Bearbeitung, Verbreitung und Wiedergabe in jeglichem Medium und Forma Bearbeitung, Verbreitung und Wiedergabe in jeglichem Medium und Fon
erlaubt, sofern Sie den/die ursprünglichen Autor(en) und die Quelle ordnungsgemäß nennen, einen Link zur Creative Commons Lizenz beifügen und angeben, ob Änderungen vorgenommen wurden. Die in diesem Artikel enthaltenen Bilder und sonstiges Drittmaterial unterliegen ebenfalls der genannten Creative Commons Lizenz, sofern sich aus der Abbildungslegen nichts anderes ergibt. Sofern das betreffende Material nicht unter der nach gesetzlichen Vorschriften erlaubt ist, ist für die oben aufgeführten nach gesetzlichen Vorschriften erlaubt ist, ist fur die oben aufgefuhr Rechteinhabers einzuholen. Weitere Details zur Lizenz entnehmen Sie bitte der Lizenzinformation auf http://creativecommons.org/licenses/by/4.0/deedde.

Korrespondenzadresse:

Prof. Dr. Jan Pielage

Abteilung Zoologie-Neurobiologie

Technische Universität Kaiserslautern

Erwin-Schrödinger-Straße 13

D-67663 Kaiserslautern

pielage@bio.uni-kl.de

www.bio.uni-kl.de/zoologie

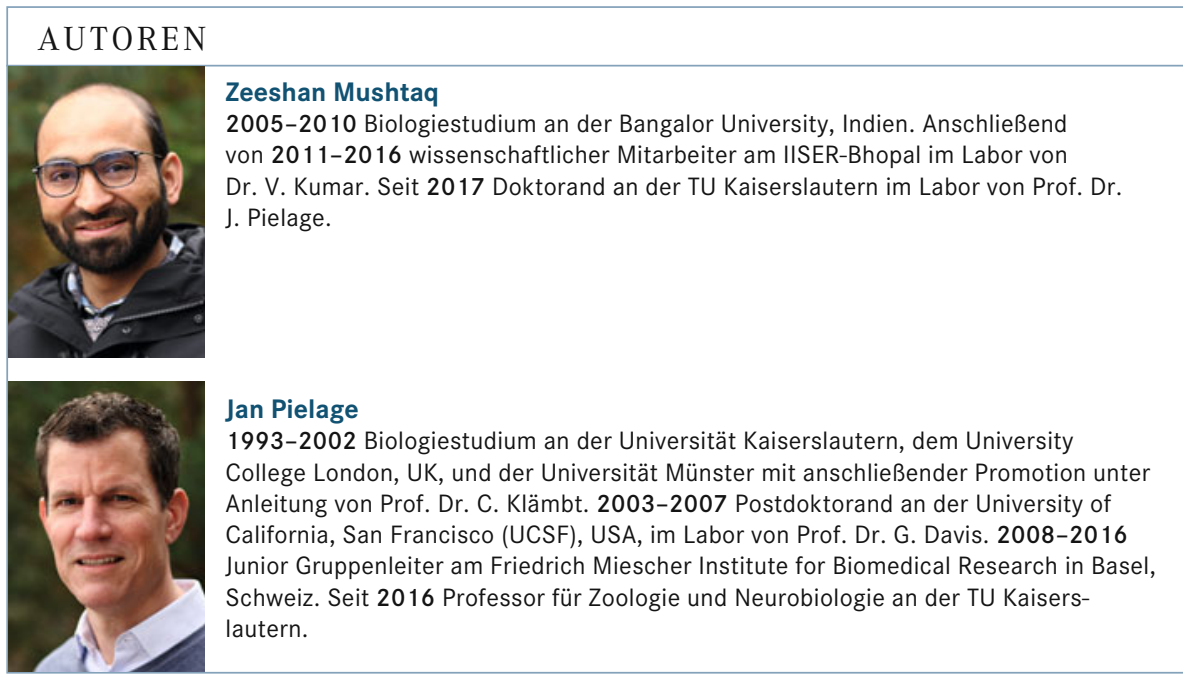

